\title{
Cardiac ablation
}

\author{
Kelly Ratheal MD, Anurag Singh MD
}

\begin{abstract}
Cardiac ablation is a procedure that uses either radiofrequency or cryothermal energy to destroy cells in the heart to terminate and/or prevent arrhythmias. The indications for cardiac catheter ablation include refractory, symptomatic arrhythmias, with more specific guidelines for atrial fibrillation in particular. The ablation procedure itself involves mapping the arrhythmia and destruction of the aberrant pathway in an effort to permanently prevent the arrhythmia. There are many types of arrhythmias, and they require individualized approaches to ablation based on their innately different electrical pathways. Ablation of arrhythmias, such as Wolff-Parkinson-White syndrome, AV nodal reentrant tachycardia, and atrial-fibrillation, is discussed in this review. Ablation has a high success rate overall and minimal complication rates, leading to improved quality of life in many patients.
\end{abstract}

Key words: catheter ablation, atrial fibrillation, arrhythmia, mapping, radiofrequency

\section{INTRODUCTION}

Cardiac ablation is a procedure used to destroy cells in the heart to terminate and/or prevent arrhythmias. The electrical signals associated with cardiac arrhythmias interfere with the sinus rhythm of a normally functioning heart, and, if not treated, cause symptoms ranging from fatigue to palpitations, syncope, and in severe cases death. The rationale for ablation therapy considers the limited long-term effectiveness of antiarrhythmic drugs and the side-effects associated with these drugs, which limit their use in many patients. ${ }^{1}$ The rationale for cardiac ablation is also based on anatomical principles. Particular arrhythmias originate at specific anatomic sites in the

Cooresponding Author: Kelly Ratheal MD

Author Contact Information: Kelly.ratheal@ttuhsc.edu DOI: $10.12746 /$ swrccc2016.0413.167 heart, which makes ablation of these locations a direct and logical approach to terminate these arrhythmias. Cardiac ablation can be surgical or non-surgical. This discussion will focus on non-surgical ablation, otherwise known as cardiac catheter ablation.

\section{INDICATIONS}

There are several indications for cardiac catheter ablation. It is commonly used for arrhythmias that are symptomatic, recurrent, and refractory to medical therapy. In addition, persistent tachyarrhythmia resulting in left ventricular systolic dysfunction and recurrent ventricular arrhythmias resulting in frequent shocks from implanted cardioverter-defibrillators are indications for catheter ablation. ${ }^{2-6}$ Although there are no absolute contraindications for ablation, relative contraindications include unstable angina, bacteremia/septicemia, and acute decompensated $\mathrm{CHF}^{7}$ 
Precise recommendations exist for atrial fibrillation. Ablation is recommended in patients with symptomatic, paroxysmal atrial fibrillation who have all of the following characteristics: failed treatment with one or more class I or III antiarrhythmic drugs, normal or mildly dilated left atria, normal or mildly reduced left ventricular function, and no severe pulmonary disease (ACC/AHA Class I, Level A). . Catheter ablation is also recommended in symptomatic patients with atrial fibrillation and Wolff-ParkinsonWhite (WPW) pre-excitation syndrome, especially if they have syncope due to rapid heart rates or short bypass tract refractory periods (ACC/AHA Class I, Level B). ${ }^{1}$ Catheter ablation is a reasonable choice in patients with symptomatic persistent atrial fibrillation with contraindications to or symptoms despite the use of more than one class I or III antiarrhythmic medications (ACC/AHA Class Ila, Level A). ${ }^{1}$ It is also reasonable to attempt ablation in recurrent symptomatic paroxysmal atrial fibrillation patients as initial treatment prior to antiarrhythmic drug therapy (ACC/AHA Class Ila, Level B).

\section{TECHNIQUE}

Cardiac ablation involves the insertion of a catheter into the selected vessel (neck, arm, or groin) and advancing it into the heart. To ablate an arrhythmia, it is necessary to identify the origin of the aberrant electric signal, which is achieved by cardiac mapping. Endocardial mapping is the simplest form of mapping, and this involves moving a catheter to various points of interest on the endocardium to measure local activation and provide spatial information. For this technique to be successful, the arrhythmia being mapped must be relatively stable, and the patient must be able to tolerate the sustained arrhythmia. Epicardial mapping may be useful in patients in whom an endocardial approach is unsuccessful. Three-dimensional electroanatomical mapping is integrated with other imaging modalities, such as a three-dimensional computed tomography or magnetic resonance image of the heart and provides the ability to "revisit" a particular site identified previously during the study, even if the tachycardia is no longer present or induc- ible..$^{8-10}$ Noncontact mapping uses a multielectrode array mounted on a balloon tipped catheter. This allows recreation of the endocardial activation sequence from simultaneously acquired multiple data points over a few (theoretically one) tachycardia beats. ${ }^{11-13}$ This eliminates the need for prolonged tachycardia episodes that patients may tolerate poorly. Mapping of the arrhythmia prior to performing an ablation is not required for all arrhythmias as some have a known course that allows for anatomic localization.

The two major tools used for ablation are radiofrequency and cryothermal energy. Radiofrequency is a low voltage high frequency electrical energy, which produces controlled focal tissue destruction. Since radiofrequency ablation does not directly stimulate nerves or myocardium, the procedure is usually relatively painless and general anesthesia is not necessary. Cryothermal ablation uses cold energy and offers the benefit of potentially reversible injury. If mild cooling has the intended effect, more profound freezing can inflict permanent tissue damage. If limited cooling does not have the desired effect, the tissue is allowed to thaw without permanent damage..$^{14-17}$ Certain types of arrhythmias are more amenable to ablation and have a higher success rate than others. These include supraventricular tachycardias, such as AV nodal reentrant tachycardia (AVNRT), Wolff-Parkinson-White syndrome producing AVRT (atrioventricular reentrant tachycardia), atrial tachycardia, atrial fibrillation, atrial flutter, and ventricular tachycardia. ${ }^{18-21}$ In typical or common type I atrial flutter, the isthmus of tissue between the tricuspid annulus (TA) and inferior vena cava (IVC) is an obligatory route. The goal of ablation is to create a line of ablation points producing a complete bidirectional electrical conduction block between the TA and IVC.

Ablation of atrial fibrillation is often anatomically based. The origin of atrial fibrillation is frequently within the sleeve of electrically active syncytial myocardium extending from the left atrium to the entrance of the pulmonary veins. ${ }^{1}$ Knowing this, placement of circumferential ablation lesions around pulmonary vein ostia creates electrical isolation and may cure atrial fibrillation. Wolff-Parkinson-White syndrome is 
defined by tachycardia associated with an AV reentrant accessory pathway. Ablation of this arrhythmia requires destruction of the accessory pathway. ${ }^{25} \mathrm{AV}$ nodal reentrant tachycardia (AVNRT) is more complex as it is usually caused by reentry using two or more accessory pathways in close proximity to the AV node. One of these conducts slowly (slow pathway) but recovers excitability rapidly and the other conducts rapidly (fast pathway) but recovers slowly setting up a closed loop incessant circuit. Ablation of the slow pathway is needed to cure the arrhythmia. ${ }^{26}$ In rare cases the AV node may be injured during the ablation and a pacemaker implantation is required. The patient then is dependent on the implanted pacemaker for life.

\section{EVALUATION OF EFFICACY - SHORT AND LONG TERM OUTCOMES}

Most of the data concerning efficacy of cardiac ablation comes from radiofrequency ablation studies attempting to cure atrial fibrillation. Although significant heterogeneity is seen with single procedures, long term freedom from atrial arrhythmia can be achieved in most patients, but multiple (typically two) ablations may be required. ${ }^{24}$ Ablation tends to be more successful for paroxysmal atrial fibrillation (episodes lasting 7 days or less) than for persistent atrial fibrillation (episodes lasting greater than 7 days). ${ }^{27}$ In patients with paroxysmal atrial fibrillation, ablation is successful $70 \%-80 \%$ of the time. In persistent atrial fibrillation, ablation is successful only $50 \%$ of the time. Ablation is less likely to work the longer a patient has persistent atrial fibrillation and in patients with structural heart disease. ${ }^{27}$

After catheter ablation, medications and follow-up are essential components of management. Anticoagulation/antithrombotic therapy for 2-3 months post procedure is recommended. Corticosteroids for 3 days after radiofrequency ablation decrease short and long-term atrial fibrillation recurrence (Level 2 evidence). ${ }^{1}$ Antiarrhythmic medication for 6 weeks may reduce the incidence of atrial fibrillation related ad- verse events at 6 weeks (Level 2) but not at 6 months (Level 3). ${ }^{1}$ Early recurrence (within 3 months of ablation) requires repeat ablation $20 \%-40 \%$ of the time. However, since some early recurrences resolve spontaneously, repeat ablation should be deferred for at least 3 months if possible. Late recurrence ( $\geq 1$ year) occurs in $11 \%-29 \%$ of patients after a single ablation procedure, and in $7 \%-24 \%$ after repeat procedures. Predictors of late recurrence include persistent atrial fibrillation, age, the size of the left atrium, diabetes, valvular heart disease, and non-ischemic dilated cardiomyopathy. ${ }^{23}$ In the event of paroxysmal atrial fibrillation recurrence, recommendations include a 12lead electrocardiogram (ECG) at each follow-up visit, 24-hour Holter at the end of the follow-up period (e.g., 12 months), and event recording regularly and at the time of symptoms with an event monitor from the end of the 3-month blanking period to the end of follow-up (e.g., 12 months). For persistent or longstanding atrial fibrillation recurrence the recommendations are 12lead ECG at each follow-up visit, 24-hour Holter every 6 months, and symptom-driven event monitoring.

\section{COMPLICATIONS}

The overall incidence of periprocedural complications related to catheter ablation has decreased with time and experience. Based on a systematic review of 83,236 patients ages 51-77 treated with catheter ablation from 2000 to 2012, the complication rate was $4 \%$ from 2000 to 2006 and decreased to $2.6 \%$ in the patients treated between 2007 and $2012 .^{28}$ Complications of cardiac ablation can be separated into several categories. First, complications may arise due to the catheterization of vessels. These complications vary from bleeding, hematoma, thromboembolism, and infection to sepsis, pseudoaneurysm formation, and transient ischemic attack or stroke. Complications may also occur secondary to the intracardiac manipulation. These include cardiac tamponade, bundle branch block, cardiac chamber or coronary sinus perforation, fibrillation or new arrhythmias, and myocardial infarction. Additionally, phrenic nerve paralysis, complete heart block, cardiac wall rupture, pulmonary vein stenosis, radiation burns, and atrio- 
esophageal fistula can develop.

Author Affiliation: Kelly Ratheal is a resident in Internal Medicine at Texas Tech University Health Sciences Center in Lubbock, TX. Anurag Singh is an electrophysiologist in the Department of Internal Medicine at TTUHSC in Lubbock, TX.

Received: $11 / 29 / 2015$

Accepted: 01/01/2016

Reviewers: Scott Shurmur MD

Published electronically: 01/15/2016

Conflict of Interest Disclosures: None

\section{REFERENCES}

1. Epstein L, Fedorowicz Z, Oettgen P. Ablation therapy for atrial fibrillation. In DynaMed [database online]. EBSCO Information Services. http://web.a.ebscohost.com.ezproxy.ttuhsc. edu/dynamed/detail?vid=2\&sid=f8c54952-dbf5-4673-8ba8-5ad ab95f4c2c\%40sessionmgr4004\&hid $=4104 \&$ bdata $=$ JnNpdGU9 ZHluYW11ZC1saXZ1JnNjb3B1PXNpdGU\%3d\#anchor=search match_0\&AN=901111\&db=dme. Updated September 21, 2015. Accessed September 22, 2015.

2. Morady F. Radio-frequency ablation as treatment for cardiac arrhythmias. N Engl J Med 1999 Feb 18; 340(7):534-44.

3. Zipes DP, DiMarco JP, Gillette PC, et al. Guidelines for clinical intracardiac electrophysiological and catheter ablation procedures. a report of the American College of Cardiology/American Heart Association Task Force on practice guidelines. (Committee on Clinical Intracardiac Electrophysiologic and Catheter Ablation Procedures). Developed in collaboration with the North American Society of Pacing and Electrophysiology. J Am Coll Cardiol 1995 Aug; 26(2):555-73.

4. Kay GN, Epstein AE, Dailey SM, et al. Role of radiofrequency ablation in the management of supraventricular arrhythmias: experience in 760 consecutive patients. J Cardiovasc Electrophysiol 1993 Aug; 4(4):371-89.

5. Chen SA, Chiang CE, Tai CT, et al. Complications of diagnostic electrophysiologic studies and radiofrequency catheter ablation in patients with tachyarrhythmias: an eight-year survey of 3,966 consecutive procedures in a tertiary referral center. $\mathrm{Am} \mathrm{J}$ Cardiol 1996; 77(1):41-6.
6. Ganz LI, Friedman PL. Supraventricular tachycardia. $N$ Engl J Med 1995 Jan 19; 332(3):162-73.

7. Tracy CM, Akhtar M, DiMarco JP, et al. American College of Cardiology/American Heart Association 2006 update of the clinical competence statement on invasive electrophysiology studies, catheter ablation, and cardioversion: a report of the American College of Cardiology/American Heart Association/American College of Physicians Task Force on Clinical Competence and Training developed in collaboration with the Heart Rhythm Society. J Am Coll Cardiol 2006 Oct 3; 48(7):1503-17.

8. Gepstein L, Hayam G, Ben-Haim SA. A novel method for nonfluoroscopic catheter-based electroanatomical mapping of the heart. In vitro and in vivo accuracy results. Circulation 1997 Mar 18; 95(6):1611-22.

9. Ben-Haim SA, Osadchy D, Schuster I, et al. Nonfluoroscopic in vivo navigation and mapping technology. Nat Med 1996 Dec; 2(12):1393-5.

10. Pérez-Castellano N, Villacastín J, Moreno J, et al. Errors in pulmonary vein identification and ostia location in the absence of pulmonary vein imaging. Heart Rhythm 2005 Oct; 2(10):1082-9. 11. Schilling RJ, Peters NS, Davies DW. Feasibility of a noncontact catheter for endocardial mapping of human ventricular tachycardia. Circulation 1999 May 18; 99(19):2543-52.

12. Schneider MA, Ndrepepa $G$, Zrenner B, et al. Noncontact mapping-guided catheter ablation of atrial fibrillation associated with left atrial ectopy. J Cardiovasc Electrophysiol 2000 Apr; 11(4):475-9.

13. Schilling RJ, Kadish AH, Peters NS, et al. Endocardial mapping of atrial fibrillation in the human right atrium using a noncontact catheter. Eur Heart J 2000 Apr; 21(7):550-64.

14. Skanes AC, Klein G, Krahn A, et al. Cryoablation: potentials and pitfalls. J Cardiovasc Electrophysiol 2004 Oct; 15(10 Suppl):S28-34.

15. Rodriguez LM, Leunissen J, Hoekstra A, et al. Transvenous cold mapping and cryoablation of the AV node in dogs: observations of chronic lesions and comparison to those obtained using radiofrequency ablation. J Cardiovasc Electrophysiol 1998 Oct; 9(10):1055-61.

16. Skanes AC, Dubuc M, Klein GJ, et al. Cryothermal ablation of the slow pathway for the elimination of atrioventricular nodal reentrant tachycardia. Circulation 2000 Dec 5; 102(23):2856-60. 17. Rodriguez LM, Geller JC, Tse HF, et al. Acute results of transvenous cryoablation of supraventricular tachycardia (atrial fibrillation, atrial flutter, Wolff-Parkinson-White syndrome, atrioventricular nodal reentry tachycardia). J Cardiovasc Electrophysiol 2002 Nov; 13(11):1082-9.

18. Narula OS. Sinus node re-entry: a mechanism for supraventricular tachycardia. Circulation 1974 Dec; 50(6):1114-28.

19. Gomes JA, Mehta D, Langan MN. Sinus node reentrant tachycardia. Pacing Clin Electrophysiol 1995 May; 18(5 Pt 
1):1045-57.

20. Krahn AD, Yee R, Klein GJ, et al. Inappropriate sinus tachycardia: evaluation and therapy. J Cardiovasc Electrophysiol 1995 Dec; 6(12):1124-8.

21. Ueng $\mathrm{KC}$, Lee $\mathrm{SH}, \mathrm{Wu} \mathrm{DJ}$, et al. Radiofrequency catheter modification of atrioventricular junction in patients with COPD and medically refractory multifocal atrial tachycardia. Chest 2000 Jan; 117(1):52-9.

22. Cosio FG, Arribas F, Lopez-Gil M, et al. Radiofrequency ablation of atrial flutter. J Cardiovasc Electrophysiol 1996 Jan; 7(1):60-70.

23. O'Riordan M. "Sobering” long-term outcomes following ablation of atrial fibrillation. In Heartwire from Medscape. Originally published January 5, 2011. http:/www.medscape.com/ viewarticle/735306

24. Ganesan A, Shipp N, Brooks A, et al. Arrhythmia and Electrophysiology. Long-term outcomes of catheter ablation of atrial fibrillation: a systematic review and meta-analysis. $J$ Am Heart Assoc 2013; 2:e004549. Originally published March 18, 2013. Doi: 10.1161/JAHA.112.004549 http://jaha.ahajournals.org/content/2/2/e004549.full

25. Kulig J, Koplan B. Wolff-Parkinson-White syndrome and accessory pathways. Circulation 2010; 122:e480-83. doi: 10.1161/ CIRCULATIONAHA.109.929372 http:/circ.ahajournals.org/ content/122/15/e480.full

26. Nakagawa H, Jackman W. Catheter ablation of paroxysmal supraventricular tachycardia. Circulation 2007; 116:2465-78.

27. Pai R, Miller J. Catheter ablation for atrial fibrillation. http:// www.webmd.com/heart-disease/atrial-fibrillation/radiofrequency-catheter-ablation-for-atrial-fibrillation. Updated August 5, 2014. Accessed December 7, 2015.

28. Cappato R, Calkins H, Chen SA, et al. Prevalence and causes of fatal outcome in catheter ablation of atrial fibrillation. $J \mathrm{Am}$ Coll Cardiol 2009 May 12; 53(19):1798-803. 